\title{
Uma Taxonomia Ontológica para a Classificação de Estudos em Informática Médica
}

\author{
Moises Roberto de A. Mota ${ }^{1}$, Esaú A. Tavares ${ }^{1}$, Sidney Patrézio R. Campos ${ }^{1}$, \\ Natasha C. Q. Lino' \\ ${ }^{1}$ Centro de Informática - Universidade Federal da Paraíba (UFPB) \\ CEP 58059-900 - João Pessoa - PB - Brasil \\ \{esau, sidneypatrezio, moises.mota\}@di.ufpb.br, \{natasha\}@ci.ufpb.br
}

\begin{abstract}
In view of the importance that Medical Informatics has acquired through the use of Artificial Intelligence techniques in their applications, especially concerning to Knowledge Engineering and Ontologies, it is necessary to map the evolution of this area thoroughly, impartially and systematically. Therefore, we built a Systematic Mapping that aims documenting about the use of Ontologies in Medical Informatics. By the fact that we don't find a general taxonomy that defines the subfields of Medical Informatics and that allows sorting the selected studies for Systematic Mapping, this paper presents a study about the construction of a taxonomy that characterizes all subareas of Medical Informatics.
\end{abstract}

Resumo. Tendo em vista a importância que a Informática Médica adquiriu, através da utilização de técnicas de Inteligência Artificial em suas aplicações, especialmente em relação ao Engenharia do Conhecimento e Ontologias, é necessário mapear a evolução desta área de modo completamente, imparcial e sistemático. Por isso, foi desenvolvido um mapeamento sistemático que tem como objetivo documentar o uso de ontologias em Informática Médica. Por não ter sido encontrada uma taxonomia geral que defina as subáreas da Informática Médica e permita a classificação dos estudos selecionados para mapeamento sistemático, este trabalho apresenta um estudo sobre a construção de uma taxonomia que caracterize as subáreas de Informática Médica.

\section{Introdução}

Representação do Conhecimento é a área da Inteligência Artificial que lida como o conhecimento deve ser representado simbolicamente e manipulado de forma automática por programas de raciocínio, Russell e Norvig (2009). Na Ciência da Computação, Ontologia é uma técnica de Representação do Conhecimento que se refere a um artefato de engenharia constituído por um vocabulário específico usado para descrever uma determinada realidade e por uma série de suposições sobre o significado pretendido do vocabulário. Ontologias descrevem uma especificação formal sobre certo domínio, sendo um entendimento compartilhado sobre o mesmo e um modelo formal e executável por máquina.

Informática Médica surge como uma nova área de pesquisa que combina as áreas de Ciência da Computação, Tecnologias da Informação e Medicina, Silachan 
(2011); lida com o armazenamento, recuperação e uso da informação, dados e conhecimentos biomédicos para a resolução de problemas e tomadas de decisão.

Neste contexto, pesquisas em Informática Médica (IM) e Inteligência Artificial podem usufruir de inúmeras oportunidades para avançar o estado da arte em vários aspectos. Tecnologias como as Ontologias facilitam a integração de dados heterogêneos, bem como tornam possível a comunicação entre sistemas diferentes, além de tornar certos tipos de dados passíveis de processamento automático. Este tipo de recurso aplicado à Informática Médica torna possível certas operações antes impraticáveis, por exemplo: o diagnóstico médico baseado em várias fontes diferentes de dados, a digitalização de prontuários médicos, a mineração de dados em bases de clínicas, etc.

Devido ao uso crescente de Ontologias na Informática Médica, tornou-se necessária a obtenção e documentação de informações, de maneira eficiente, sobre o estado da arte do uso de Ontologias na Informática Médica, com o objetivo de promover uma visão detalhada de como as aplicações são desenvolvidas nessa área, quais seus benefícios e principais técnicas utilizadas. Assim, contribuindo para o crescimento da área, com ênfase nas suas necessidades e deficiências, evitando a criação de estudos duplicados ou não-relevantes. Dessa forma, optou-se pelo desenvolvimento de um Mapeamento Sistemático sobre o uso de Ontologias na Informática Médica.

Segundo Kitchenham (2007), o Mapeamento Sistemático é uma metodologia de pesquisa e avaliação de literatura que tem como objetivo identificar todas as pesquisas relacionadas a um tópico específico, ou seja, responder a questões mais amplas relacionadas à evolução da investigação de uma determinada área da ciência. Deste modo, tem-se uma visão geral da evolução de uma determinada área de pesquisa até o momento da construção do mapeamento. Além de documenta-la de maneira sistemática permitindo sua replicação, avaliação e posterior atualização.

O Mapeamento Sistemático (MS) é composto por três etapas, Kitchenham (2007), são elas: Etapa de Planejamento, Etapa de Condução e Etapa de Reporte. Todas essas etapas precisam ser bem documentadas, Arksey e O'Malley (2005). Os trabalhos de Jacinto (2010), Almeida (2011), Cardozo (2012), abordam que o processo para escolha, ou desenvolvimento, de uma taxonomia adequada para a classificação dos trabalhos de um MS nem sempre é detalhado, visto que, geralmente é utilizada uma taxonomia validada e bem conceituada pela comunidade acadêmica para qual é direcionado o MS. Por exemplo, no trabalho de Suassuna (2011) foi utilizado o SWEBOK, seus capítulos e sessões para classificar os estudos selecionados para o Mapeamento Sistemático.

Para o presente trabalho não foi encontrada, até o momento, uma taxonomia com os requisitos necessários. Desse modo, foi necessário o desenvolvimento de uma taxonomia que proporcione uma estruturação de dados em estudos cujo foco é o uso de Ontologias em Informática Médica.

\section{Planejamento do Mapeamento Sistemático}

Dentro da etapa de Planejamento do Mapeamento, a atividade mais importante, de acordo com Kitchenham (2007), é a criação das questões de pesquisa. As questões de pesquisa são o foco do mapeamento, e são elas o guia da pesquisa. Além disso, as questões de pesquisa atuam como um dos fatores de inclusão e exclusão na busca 
automática de estudos e trabalhos, pois apenas são considerados artigos que abordem o tema de alguma dessas questões. As questões de pesquisa que orientaram este trabalho são:

- Qual(is) o(s) principal(is) uso(s) de Ontologias em Informática Médica?

- Qual(is) o(s) benefícios do uso de Ontologias em Informática Médica?

- Quais as ontologias utilizadas em Informática Médica?

- É considerado o reuso de ontologias nos projetos de Informática Médica?

- Quais a ferramentas utilizadas para a criação e edição de Ontologias?

- Quais repositórios de Ontologias são utilizados?

- Como os trabalhos de Ontologias em Informática Médica estão sendo validados/avaliados?

O próximo passo consiste em especificar os engenhos de busca para a execução da busca automática. Os engenhos de busca apresentados na Tabela 1 foram selecionados por possuírem, segundo a literatura pesquisada, Dyba (2005), Kitchenham (2007), Travassos (2007), Brereton (2007), boa parte das pesquisas desenvolvidas no domínio da Ciência da Computação. Foram excluídos engenhos pela indisponibilidade de seus artigos, por não possuir convênio com a Universidade Federal da Paraíba ou por abordarem temas que não são de interesse desta pesquisa.

Também foram definidos periódicos para compor a busca manual, como: International Journal of Metadata, Semantics and Ontologies (Qualis B2), International Journal of Medical Informatics (Qualis A2), Journal of Biomedical Informatics (Qualis A1), Journal of the American Medical Informatics Association (Qualis A1), Medical Informatics and the internet in Medicine (Qualis B2) e Applied Ontology (Qualis B2).

Em seguida, é preciso construir uma String de Busca, Kitchenham (2007) e Travassos (2007), que deve ser expressivo para representar o conjunto de termos encontrados nas questões de pesquisa e recuperar os trabalhos relevantes para o mapeamento com precisão e cobertura.

Tabela 1. Engenhos utilizados na busca automática

\begin{tabular}{|c|c|}
\hline Engenho de busca & Endereço Web \\
\hline ACM & dl.acm.org/dl.cfm \\
\hline IEEE & ieeexplore.ieee.org/ \\
\hline Science Direct & sciencedirect.com/ \\
\hline PubMed & www.ncbi.nlm.nih.gov/pubmed \\
\hline PMC & www.ncbi.nlm.nih.gov/pmc/ \\
\hline Google Scholar & scholar.google.com.br/ \\
\hline
\end{tabular}


A String de Busca é construída a partir da concatenação dos termos encontrados nas questões de pesquisa através de operadores booleanos (“AND" e "OR"). Uma vez desenvolvida a String de Busca, vide tabela 2, é possível iniciar a pesquisa nos engenhos selecionados. A String de Busca para este trabalho em específico esta definida abaixo.

Tabela 2. String de Busca

("Knowledge Engineering" OR "Knowledge Representation" OR "Ontology Engineering") AND ("Health" OR "E-Health" OR "Electronic Health" OR "Medical Informatics" OR "Medical Systems" OR "Medical" OR "Clinical Decision Support" OR "Medical Decision Support") AND ("Ontology" OR "Ontologies")

Para auxiliar a seleção dos estudos relevantes para um Mapeamento Sistemático é preciso que existam critérios de inclusão e exclusão, Kitchenham (2007), Travassos (2007).

- Os seguintes tipos de estudos serão incluídos nesta avaliação sistemática:

- Estudos completos publicados em revistas ou conferências revisadas sobre o uso de Ontologias em Informática Médica e que respondam por uma das questões de pesquisa;

- Estudos secundários, ou seja, que dependam de estudos primários;

- Estudos teóricos com o objetivo de apresentar conceitos para o entendimento da área;

- Estudos experimentais relacionados à área;

- Apenas trabalhos escritos em língua inglesa serão considerados.

Os seguintes estudos serão excluídos:

- Estudos que não estejam claramente relacionados à área de Ontologias e Informática Médica;

- Estudos que não respondem a nenhuma das questões de pesquisa;

- Artigos duplicados, ou seja, aqueles encontrados em mais de uma fonte da busca automática e/ou manual;

- Artigos convidados, tutoriais, relatórios técnicos, teses, dissertações e relatórios de workshops que sejam incompletos;

- Estudos não disponíveis para download nos engenhos de busca definidos.

Os resultados das buscas foram os seguintes: ACM: 55 trabalhos encontrados dos quais 15 foram aceitos; IEEE: 2088 foram encontrados, 215 aceitos; Science Direct: 1712 encontrados, 255 aceitos; Google Scholar: 18300 encontrados, 13 aceitos; PubMed: 64 encontrados, 29 aceitos e PMC: 1375 encontrados, 263 aceitos.

A aplicação da string de busca nos periódicos trouxe resultados em menor número e de pequena relevância para o trabalho.

A seguir, apresenta-se o processo de desenvolvimento da taxonomia para classificação dos artigos aceitos no Mapeamento Sistemático.

\section{Estudos sobre Taxonomias}

Observando os trabalhos de Feldt et al (2008), que detalham a construção de sua própria taxonomia baseada principalmente em palavras-chaves encontradas nos resumos 
dos estudos selecionados, e Dixon et al (2007), que sugerem uma categorização dos termos existentes na literatura médica; iniciou-se a definição de uma estratégia para a construção de uma taxonomia que represente as subáreas da Informática Médica. Para a execução do presente trabalho foram pesquisadas e estudadas as taxonomias das seguintes fontes atuantes em diversas partes do mundo, são elas: European Federation for Medical Informatics (EFMI); Sociedade Brasileira de Informática em Saúde (SBIS); American Medical Informatics Association (AMIA); Associação Portuguesa de Informática Médica (APIM); Medical Informatics - Practical Guide for the Healthcare Professional, Hoyt (2009); China-Japan-Korea Joint Symposium on Medical Informatics (CJKMI); Asia Pacific Association for Medical Informatics Conference (APAMI); South African Health Informatics Association Conference (SAHIAC)

Foram considerados também termos presentes nos principais congressos, períodicos e associações, que se distribuem em diversos continentes e definem as subáreas da Informática Médica.

\subsection{Taxonomias}

Ao analisar as fontes definidas na sessão anterior, foi percebido que determinadas categorizações encontradas são específicas para região em que a associação, congresso ou conferência se destina, por exemplo, categorias como Informatics of Traditional Chinese Medicine só foram identificadas na Asia Pacific Association for Medical Informatics Conference. Também é possível notar que na taxonomia proposta pela European Federation for Medical Informatics existem categorias direcionadas para a mesma subárea da Informática Médica, mas com focos específicos, por exemplo: Knowledge discovery in biomedical databases for decision support, Intelligent interoperability and telemedicine Data and knowledge management for decision support in forensic medical disciplines, eHealth decision support systems for GPs, clinicians, nurses, health care managers and patients, Evaluation of decision support systems Diagnostic, therapeutic and prognostic decision support; ou seja, essas categorias podem ser estruturadas numa hierarquia da subárea Clinical Decision Support. Entre outras, a taxonomia da American Medical Informatics Association, apresenta este mesmo detalhe com as seguintes categorias: Clinical Research Informatics, Clinical Informatics.

A taxonomia construída neste trabalho foi desenvolvida a partir da comparação entre as diversas categorias estudadas, como veremos na sessão posterior.

\section{Análises comparativas e desenvolvimento da Taxonomia}

É importante salientar a existência de outras taxonomias que definem a Informática Médica, porém, direcionadas para contextos específicos, Dixon (2007), como as propostas pelo HIMSS (Healthcare Information Menagement System Society) e pela MESH (Medical Subject Headings).

Segundo Dixon (2007), a taxonomia proposta pelo HIMSS aborda uma parte das principais subáreas da Informática Médica, porém, é estritamente direcionada para sistemas de prontuários médicos eletrônicos, desconsiderando certas aplicações da informática na medicina, como telemedicina e o compartilhamento de informações em saúde. Portanto, não foi considerada adequada para classificar os trabalhos selecionados para o Mapeamento Sistemático, uma vez que a proposta é o desenvolvimento de uma taxonomia geral que represente as principais subáreas existentes para esta grande área 
da ciência. Posteriormente esta hierarquia de termos foi atualizada e expandida pelo MEDLINE, incluindo a terminologia "tecnologias da informação em saúde". Entretanto, na hierarquia proposta pelo MESH há falta de diversos conceitos da Informática Médica principalmente no que diz respeito à implementação de prontuário eletrônico em saúde.

\subsection{Metodologia}

Conforme citado na sessão anterior, a taxonomia construída foi desenvolvida utilizando como base os estudos de Dixon (2007), para tanto, foi realizada uma comparação entre os termos presentes nas taxonomias estudadas. Posteriormente, foram selecionados os termos semelhantes ou correlacionados entre as taxonomias, de acordo com sua definição nas fontes citadas anteriormente, Feldt (2008), Richmond (2003). Uma vez selecionados, os mesmos foram agregados para a construção de uma única categoria geral. Por exemplo, os seguintes termos: Prontuário Eletrônico do Paciente (SBIS), Electronic Medical Records/Electronic Health Records (CJKMI), foram agregados ao termo Electronic Health Records, Hoyt (2009), por considerarmos este como o termo mais abrangente e que poderia classificar qualquer trabalho direcionado ao desenvolvimento de Registros Eletrônicos aplicados a Saúde.

\subsection{Resultados da Análise Comparativa}

Categorias como Electronic Prescribing e Mobile Technology encontradas em Hoyt, (2009) foram aglutinadas num só termo, Consumer Health Informatics. Este novo termo reúne todo estudo que desenvolva produtos comerciais, ou não, voltados para pacientes. Esta decisão está relacionada à constatação durante a pesquisa de que trabalhos nestas áreas são desenvolvidos para esta finalidade.

Internet em Saúde (SBIS), Networks [Hoyt 2009], Online Medical Resources [Hoyt 2009], Search Engines [Hoyt 2009], Internet of Things (APAMI) e Cloud Computing (APAMI) foram reunidas na categoria Online Medical Resources. Dessa forma, os estudos relacionados aos usos de recursos web na Informática Médica são classificados nesta categoria, bem como serviços online.

As categorias Formalization of knowledge, ontologies, clinical guidelines and standards of health care (EFMI), Standard in Health Informatics and cross-language solution (CJKMI), Standards in Medical Informatics (APAMI) e Padronização da Informação em Saúde (SBIS) foram aglutinadas no termo Standards in Medical Informatics. Os estudos aqui classificados são direcionados para definição de padrões de abrangência mundial para a aplicação de ontologias em Informática Médica.

Information Technology Infrastructure (SAHIAC), Privacy, Security, and Confidentiality Issues in Medical Information (APAMI), Medical safety management (CJKMI), Human Resources Management (SAHIAC) e Integrated Practice Management Systems [Hoyt 2009] foram agrupados na categoria Infrastructure of Medical Information Systems, direcionada para estudos que desenvolvem solução estrutural para sistemas de Informática Médica baseados em ontologias.

Intelligent interoperability and telemedicine(EFMI) e Health Information Technology Interoperability [Hoyt 2009] foram resumidas na categoria Health Management Information Systems. Esta Categoria reunirá estudos direcionados para o desenvolvimento de sistemas de Informática Médica baseados em ontologias. 
Pay for Performance (P4P) [Hoyt 2009] e Financial management for improved health service delivery (APAMI) foram reunidas no termo Financial management for improved health service delivery, cujo objetivo é reunir os trabalhos voltados para a administração de recursos financeiros para o desenvolvimento de sistemas de Informática Médica.

O termo Informatics of Traditional Chinese Medicine foi transformado em uma categoria exclusiva, tendo em vista a especificidade dos estudos desenvolvidos relacionado a este termo encontrado entre os estudos selecionados para compor o Mapeamento Sistemático.

\section{Resultados e Discussão}

Foram 23 termos considerados ideais para classificação dos estudos selecionados para o Mapeamento Sistemático. Acredita-se que esta taxonomia abrange todas as subáreas da Informática Médica, desde as mais gerais, como Telemedicine, até as mais específicas, como Nursing Informatics. O processo de desenvolvimento desta taxonomia foi baseado nos trabalhos de Dixon (2007) e Feldt (2008), de modo semelhante a estes estudos, foram selecionados, criados e agrupados termos de diversas fontes de acordo com a necessidade para o desenvolvimento do Mapeamento Sistemático.

$\mathrm{Na}$ Tabela 3 foram definidos os termos da taxonomia, baseadas nas definições de Hoyt (2009), visto que foi a única definição formal das subáreas da Informática Médica as quais tivemos acesso:

Tabela 3. Taxonomia Final e Definição dos termos

\begin{tabular}{|c|c|}
\hline Termos da Taxonomia & Definição \\
\hline Applications of Bioinformatics in Medical Informatics & $\begin{array}{l}\text { Bioinformática é uma área da ciência direcionada } \\
\text { para aplicação de tecnologias informáticas em } \\
\text { biologia, especificamente em genética. }\end{array}$ \\
\hline $\begin{array}{c}\text { Applications of Knowledge Engineering in Medical } \\
\text { Informatics }\end{array}$ & $\begin{array}{c}\text { O desenvolvimento de ontologias é uma técnica de } \\
\text { engenharia do conhecimento, e por isso essa } \\
\text { categoria é reservada para todos os estudos que } \\
\text { propõem a criação de ontologias, bem com o } \\
\text { desenvolvimento de metodologias para validações } \\
\text { e análises das mesmas. }\end{array}$ \\
\hline Artificial Intelligence in Medicine and Its Applications & $\begin{array}{c}\text { Categoria direcionada para estudos que propõem a } \\
\text { aplicação de técnicas de Inteligência Artificial em } \\
\text { sistema na Informática Médica. }\end{array}$ \\
\hline Biomedical Eletronic and Robótics Devices & $\begin{array}{l}\text { Esta subárea da Informática Médica tem como } \\
\text { objetivo o desenvolvimento de sistemas } \\
\text { biomecânicos ou robóticos que proporcionem } \\
\text { melhor qualidade de vida do paciente. }\end{array}$ \\
\hline Clinical Informatics & $\begin{array}{l}\text { Consiste em uma das diversas aplicações da } \\
\text { Informática Médica. Ou seja, na criação, } \\
\text { gerenciamento, compartilhamento e aplicação de } \\
\text { guias de práticas clínicas para tratamento de } \\
\text { determinadas doenças }\end{array}$ \\
\hline
\end{tabular}




\begin{tabular}{|c|c|}
\hline Consumer Health Informatics & $\begin{array}{c}\text { Esta subárea é voltada para o desenvolvimento de } \\
\text { aplicações e serviços móveis direcionados para o } \\
\text { consumidor. }\end{array}$ \\
\hline Decision Support Systems & $\begin{array}{c}\text { Qualquer software projetado para ajudar } \\
\text { diretamente na tomada de decisão clínica em que } \\
\text { as características de cada paciente são } \\
\text { compatíveis com uma base de conhecimento } \\
\text { informatizada. }\end{array}$ \\
\hline Disease Management and Disease Registries & $\begin{array}{c}\text { O principal objetivo desta subárea é auxiliar na } \\
\text { gestão de pacientes crônicos através da utilização } \\
\text { dos recursos aqui apresentados. }\end{array}$ \\
\hline Electronic Health Records & $\begin{array}{c}\text { Conhecidos como prontuários eletrônicos, pode ser } \\
\text { considerado o principal foco do desenvolvimento } \\
\text { da informática médica. }\end{array}$ \\
\hline Evidence Based Medicine & $\begin{array}{l}\text { Consiste no auxilio tecnológico a avaliação das } \\
\text { subáreas da medicina baseada na busca por } \\
\text { evidências. Ou seja, a avaliação da qualidade dos } \\
\text { estudos nas diversas áreas da medicina }\end{array}$ \\
\hline $\begin{array}{l}\text { Financial management for improved health service } \\
\text { delivery }\end{array}$ & $\begin{array}{c}\text { Esta subárea consiste na definição da tributação e } \\
\text { o retorno financeiro aos clínicos por aplicações na } \\
\text { área clínica, ou médica. }\end{array}$ \\
\hline Health informatics education & $\begin{array}{c}\text { Consiste em estudos que utilizam ontologias com o } \\
\text { objetivos educacionais direcionados para } \\
\text { Informática Médica. }\end{array}$ \\
\hline Health Management Information Systems & $\begin{array}{l}\text { Categoria direcionada para estudos cujo foco é o } \\
\text { desenvolvimento e gerenciamento de sistemas } \\
\text { informação na área de Informática Médica. }\end{array}$ \\
\hline Informatics of Traditional Chinese Medicine & $\begin{array}{l}\text { Categoria reservada para a medicina tracional } \\
\text { chinesa. }\end{array}$ \\
\hline Infrastructure of Medical Information Systems & $\begin{array}{c}\text { Esta categoria é direcionada para estudos que } \\
\text { propõe melhorias nas infra-estruturas dos sistemas } \\
\text { em Informática Médica. }\end{array}$ \\
\hline $\begin{array}{l}\text { Medical Data Representation, Mining, Visualization, } \\
\text { and Sharing }\end{array}$ & $\begin{array}{l}\text { Consiste no desenvolvimento de técnicas que } \\
\text { permitam o aprimoramento das tecnologias atuais } \\
\text { de representação, mineração, visualização e } \\
\text { compartilhamento de dados médicos. }\end{array}$ \\
\hline Medical Signal and Image Processings & $\begin{array}{l}\text { Subárea voltada para o processamento e análise } \\
\text { de sinais e imagens médicas, além de abranger a } \\
\text { transmissão, armazenamento e formato de dados } \\
\text { desta natureza. }\end{array}$ \\
\hline Nursing Informatics & $\begin{array}{c}\text { Esta categoria é reservada para estudos voltados } \\
\text { para o auxílio de profissionais da área de } \\
\text { enfermagem. }\end{array}$ \\
\hline
\end{tabular}




\begin{tabular}{|c|c|}
\hline Online Medical Resources & $\begin{array}{c}\text { Consiste no desenvolvimento de bases de dados } \\
\text { de informações médicas confiáveis, de modo que } \\
\text { possam ser consultados para finalidades como } \\
\text { educação, recuperação de informação por médicos } \\
\text { e pacientes, entre outros. }\end{array}$ \\
\hline Pacient Informatics & $\begin{array}{c}\text { Estudos cujo objetivo é melhorar o tratamento de } \\
\text { pacientes em hospitais crônicos e terminais, } \\
\text { inclusive evitando erros médicos. }\end{array}$ \\
\hline Public Health Informatics & $\begin{array}{c}\text { Subárea direcionada para uso de Informática } \\
\text { Médica direcionada para as populações e não } \\
\text { indivíduos. }\end{array}$ \\
\hline Standards in Medical Informatics & $\begin{array}{c}\text { A definição de padrões para a área da Informática } \\
\text { Médica é importante para a interoperabilidade de } \\
\text { sistemas diferentes. }\end{array}$ \\
\hline Telemedicine & $\begin{array}{c}\text { Consiste na transferência de dados administrativos } \\
\text { relacionado a instituições de saúde e a } \\
\text { monitoração remota de pacientes respectivamente. }\end{array}$ \\
\hline
\end{tabular}

A validação da taxonomia ocorreu na etapa da análise qualitativa, na qual, foi realizada a leitura de todos os artigos selecionados para o Mapeamento Sistemático com o objetivo de identificar suas contribuições e classificar sua(s) área(s) de atuação. Dessa maneira, pode-se identificar que o intuito da taxonomia abranger todos os trabalhos selecionados para o MS foi atingido. No entanto, é importante deixar claro que todo o processo de desenvolvimento de um Mapeamento Sistemático evolui e se adapta conforme as necessidades apresentadas durante o estudo, deste modo, esta taxonomia ainda poderá ser modificada se for identificada esta necessidade.

\subsection{Taxonomia Ontológica}

Com base na análise das terminologias estudadas e na taxonomia final, também foi desenvolvido o protótipo de uma Taxonomia Ontológica, como pode ser visto na Figura 1 .

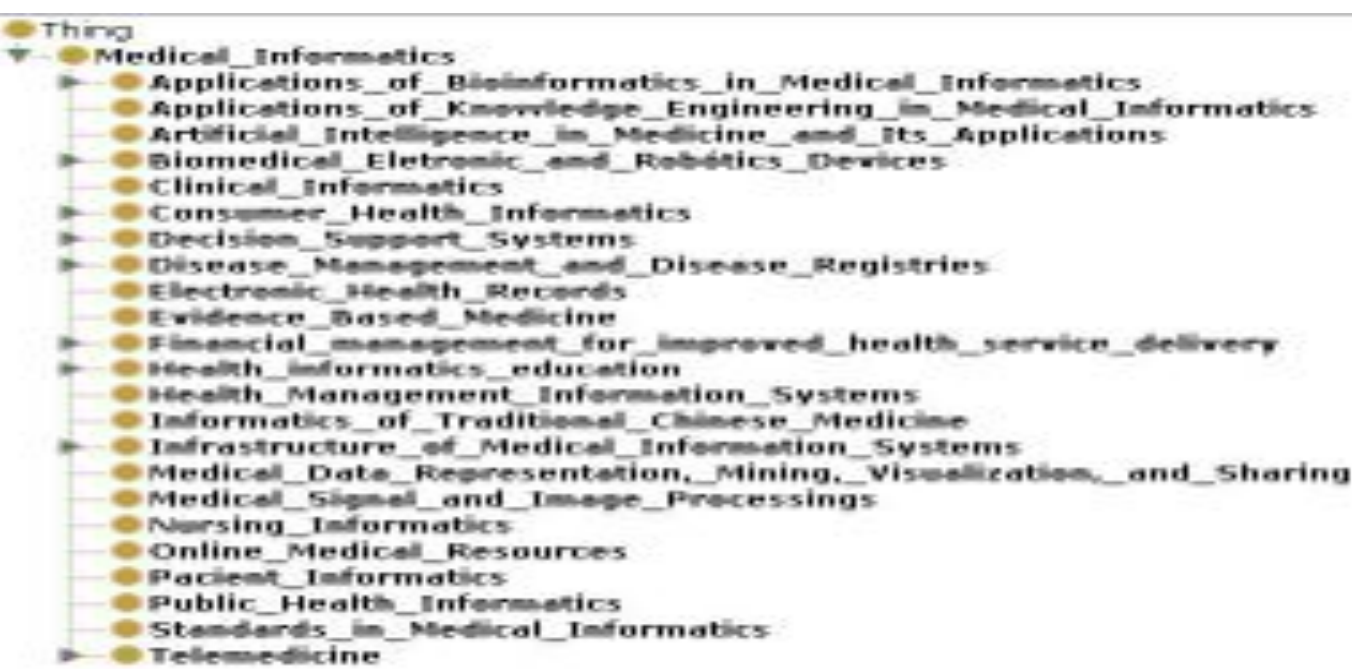

Figura 1. Taxonomia Ontológica desenvolvida no presente trabalho 
Uma taxonomia serve para classificar informações em uma hierarquia (árvore) utilizando o relacionamento pai-filho (generalização ou "tipo-de"). Uma Ontologia permite atribuir características a cada termo existente na taxonomia, fazendo uma distinção clara entre suas definições, Breitman (2010). Dessa maneira, nenhum estudo poderá ser agrupado, classificado ou relacionado erroneamente com algum termo incompatível com suas características. Portanto, através do desenvolvimento da Taxonomia Ontológica, é possível um compartilhamento e organização mais eficientes de informações presentes em bases de dados relacionadas a Informática Médica. Por ser concebida em OWL, de acordo com os padrões do W3C, sua integração com projetos como os propostos por Borges (2011) e Kamura (2011) bem como com ferramentas de buscas baseadas em tecnologias semânticas, além de ser de grande utilidade para o desenvolvimento da Web Semântica, Hausenblas (2010).

\section{Conclusões}

O desenvolvimento de uma taxonomia que defina as principais subáreas da Informática Médica para a classificação de estudos de um Mapeamento Sistemático sobre Ontologias nesta área se torna mais expressivo, uma vez que o trabalho avançou no sentido do desenvolvimento de uma Taxonomia Ontológica, além de cumprir com seu objetivo inicial. Deste modo os resultados foram além do pretendido. Uma taxonomia que categorize a Informática Médica em subáreas é importante para estruturação de bases de dados relacionadas a área, já que não foi encontrada até o momento uma que se adequasse a necessidade deste estudo, bem como fosse abrangente o suficiente para permitir a classificação de estudos concentrados em áreas inovadoras e específicas que as terminologias utilizadas como base não permitiram.

No mais, a Taxonomia Ontológica não é apenas importante para este trabalho e sua divulgação, como também para o compartilhamento e estruturação de informações na web. Por ser concebida com base em tecnologias semânticas e dentro dos padrões definidos pela $\mathrm{W} 3 \mathrm{C}$, é possível uma fácil integração com projetos como os citados por Heath (2011), permitindo também a integração de diferentes bases de dados distribuídas pela web, Hausenblas (2010).

\section{Perspectivas futuras}

O principal objetivo deste estudo foi o desenvolvimento de uma taxonomia que represente todas as subáreas da Informática Médica, para a classificação dos estudos selecionados para o Mapeamento Sistemático sobre o uso de Ontologias em Informática Médica que se encontra em desenvolvimento.

Após a conclusão do Mapeamento Sistemático, a eficácia da taxonomia, aqui proposta, será validada e posteriormente publicada, visando sua avaliação diante da comunidade científica relacionada, permitindo que outros tipos de estudos sejam desenvolvidos utilizando esta taxonomia como uma das suas referências.

Com a classificação dos estudos, da área de Informática Médica, de acordo com taxonomia proposta e após a finalização do Mapeamento Sistemático, pode-se identificar quais as subáreas da Informática Médica com maior desenvolvimento, e quais as que se beneficiam mais com o uso das tecnologias semânticas, como as 
Ontologias. Contribuindo, deste modo, para o desenvolvimento igual da área através da demonstração das suas necessidades e lacunas.

Pretendemos também validar, estender e refinar a Taxonomia Ontológica, aqui desenvolvida, para que a mesma permita a estruturação de bases de dados de estudos relacionados à Informática Médica e o compartilhamento de conhecimento mais eficiente através de aplicações que utilizem tecnologias semânticas Hausenblas (2010) e Heath (2011).

\section{Referências}

Almeida, A. T. (2011). Um Mapeamento Sistemático de Mecanismos para Guiar Estudos Empíricos em Engenharia de Software. Dissertação (Mestrado em Ciências da Computação). Universidade Federal de Pernambuco. Recife, Pernambuco.

Arksey, H and O’Malley, L. (2005). Scoping Studies: towards a methodological framework. International Journal of Social Research Methodology, vol. 8, n. 1, 19-32.

Borges, M. R. S., Campos, M. L. M., Cordeiro, K. F. and Marino, T. (2011). Use of Linked Data in the Design of Information Infrastructure for Collaborative Emergency Management System. In Proceedings of 15th International Conference on Computer Supported Cooperative Work in Design, 764-771.

Breitman, K, K. (2010). Web Semântica: A Internet do Futuro. LTC - Livros Técnicos e Científicos Ltda. Rio de Janeiro, Brasil.

Brereton, P., Kitchenham, B. A., Budgen, D. T., Mohamed, M. and Mohamed, K. (2007). Lessons from applying the systematic literature review process within the software engineering domain. JSS 80, 571-583.

Cardozo, E. S. F. (2012). Mapeamento Sistemático sobre o uso do Auto-Gerenciamento em Equipes de Desenvolvimento de Software. Dissertação (Mestrado em Ciências da Computação). Centro de Informática. Universidade Federal de Pernambuco. Recife, Pernambuco.

Dixon, B. E., McGowan, J. J. and Zafar, A. (2007). Development of a Taxonomy for Health Information Technology. In Proceedings of World Congress on Medical and Health Informatics,Australia.

Dyba, T., Jorgensen, M. and Kintchenham , B. (2005). Evidence-based software engineering for practitioners. IEEE Software, 58-65.

Feldt, R., Petersen, K., Mattsson, M. and Mujtaba, S. (2008). Systematic Mapping Studies in Software Engineering. In Proceedings of 12th International Conference on Evaluation and Assessment in Software Engineering, Bari, Italy, 71-80.

Hausenblas, M. and Karnstedt, M. (2010). Understanding Linked Open Data as a WebScale Database .In Proceedings of Second International Conference on Advances in Databases, Knowledge, and Data Applications, 56-61.

Heath, T. (2011); Linked Data - Welcome to the Data Network. IEEE Internet Computing, vol. 11, 70-73.

Hoyt, R. E., Sutton, M., and Yoshihashi, A. (2009). Medical Informatics - Practical Guide for the Healthcare Professional. $3^{\text {a }}$ Edição, University of West Florida, School of Allied Health and Life Sciences. Lulu.com. 
Jacinto, S. S. (2010). Um Mapeamento Sistemático de Pesquisa sobre a Influência da Personalidade na Engenharia de Software. Dissertação (Mestrado em Ciências da Computação). Centro de Informática. Universidade Federal de Pernambuco. Recife, Pernambuco.

Kamura, T., Kato, F., Ohmukai, I., Takahashi, T., Takeda, H. and Ueda, H. (2011). Study support and integration of cultural information resources with Linked Data. In Proceedings of Second International Conference on Culture and Computing, 177 - 178.

Kitchenham, B. (2007). Guidelines for performing Systematic Literature Reviews in Sofware Engineering. Vol 2.3 EBSB Technical Report, EBSE-2007-01.

Richmond, B. (2003). D. H. The Truth About Taxonomies. Info Mgmt. (Mar/Apr. 2003), 44-53.

Russell, S. and Norvig, P. (2009). Artificial Intelligence: A Modern Approach. 3rd Edition. New Jersey: Prentice Hall.

Silachan, K. (2011). Tantatsanawong, P. Domain Ontology Health Informatics Service From Text Medical Data Classification. Department of Computing, Faculty of Science, Silpakorn University. Nakornpratom, Thailand. In Proceedings of Annual SRII Global Conference.

Suassuna, M. (2011). Mapeamento Sistemático sobre Replicação de Estudos Empíricos em Engenharia de Software. Dissertação (Mestrado em Ciências da Computação). Centro de Informática. Universidade Federal de Pernambuco. Recife, Pernambuco.

Travassos, G. and Biolchini J. (2007). Revisões Sistemáticas Aplicadas a Engenharia de Software. In Proceedings of XXI SBES - Brazilian Symposium on Software Engineering, João Pessoa, PB, Brasil. 